\title{
Interval Valued Intuitionistic Fuzzy Gaussian Membership Function: A Novel Extension
}

\author{
Janani Bharatraj ${ }^{(\mathbb{\square})}(\mathbb{0})$ \\ Hindustan Institute of Technology and Science, Chennai 603103, India \\ jananichari@gmail.com
}

\begin{abstract}
The Interval-valued intuitionistic fuzzy sets (IVIFSs) have long been used to model vagueness and have been used in decision making, pattern recognition, image processing, and other applications. In this study, IVIFSs are defined using Gaussian membership functions (GMFs), and new measures of the distance, the overlap, and the angle between two sets are developed. The proposed methodology is used to determine the similarities between test subjects in genetic brain profiling.
\end{abstract}

Keywords: Bhattacharya angle $\cdot$ Bhattacharya distance $\cdot$ Gaussian membership function - Genetic brain profiling - Interval valued intuitionistic fuzzy sets

\section{Introduction}

Fuzzy set theory and its extensions have gained popularity in various areas of science, engineering, and technology since their inception in 1967. Fuzzy set theory and its extensions are founded on three main concepts, namely, imprecision, uncertainty, and vagueness. Uncertainty and vagueness form an integral part of type- 1 and type- 2 fuzzy sets, interval-valued fuzzy sets (IVFSs), and intuitionistic fuzzy sets (IFSs). Atanassov combined IVFSs and IFSs to create a more effective method of modelling vagueness known as interval-valued intuitionistic fuzzy sets (IVIFSs). Since its inception, IVIFSs have been applied to various areas of study like decision making and pattern recognition techniques. Distance measures on IVIFSs based on all three degrees of freedom have been studied for use in pattern recognition. The present study explores IVIFSs using an alternative membership function. The remainder of the paper is organized as follows. Section 2 provides a literature review of similarity measures on IVIFSs with applications in pattern recognition. Section 3 identifies areas for improvement in the existing methods. Section 4 defines certain properties required for developing an extended version of the IVIFS with Gaussian membership functions (IVIFS-GMFs). Section 5 introduces IVIFS-GMFs and defines a distance, or similarity, measure for IVIFS-GMFs, and a simple example of pattern recognition using IVIFS-GMFs is presented in Sect. 6. 


\section{Literature Review}

Yuan and Li [4] developed five-valued fuzzy sets that constituted the threshold sets of IVIFSs. The properties of these sets were found to be consistent with the properties of fuzzy sets and intuitionistic fuzzy sets. Yuan et al. [8] further developed cut sets on IVIFSs and derived representation and decomposition theorems for interval-valued and intuitionistic fuzzy sets. Adak et al. [1] introduced interval cut sets of generalized IVIFSs and studied their properties using fuzzy arithmetic. Taking a geometric interpretation of IVIFSs, Zeshui [9] defined distance measures such as the Hamming distance and the Euclidean distance with the use of a Hausdorff metric. Weighting factors on the elements were defined, and weighted distance measures between IVIFSs were developed. An example problem, the classification of building materials, was presented to demonstrate the approach. Zhang et al. [11] constructed a novel pattern recognition approach for measuring the degree of similarity between IVIFSs by choosing a different weight for each feature according to its dissimilarity with other features. Zhang et al. [10] constructed a new pattern recognition approach with IVIFSs by defining separate Hausdorff metrics for lower membership functions and upper membership functions and deriving different similarity measures for each of the functions. The effect of using both the measures in combination was then tested on a pattern recognition problem. Wei et al. [6] proposed an entropy measure on IVIFSs that generalized the entropy measures on IFSs. New similarity measures were constructed using the new entropy measures, and the effectiveness of these measures were demonstrated using a pattern recognition example. Singh [5] investigated a cosine similarity measure for IVIFSs. An example of pattern recognition in medical diagnostics was used to test the effectiveness of the cosine measure. It was found that the new cosine similarity measure provided better results than distance-based similarity measures. Zhang et al. [12] introduced the dynamic IVIFS, which was shown to be more accurate in a medical diagnostics and decision-making example. The technique was more comprehensive and more flexible than IFS and IVIFS methods. Meng and Chen [4] defined an entropy measure using Shapley-weighted similarity measures that included the interdependencies of the elements in a set. This method was applied to a pattern recognition problem with the multi-criteria decision-making technique.

\section{Research Statement and Objectives}

The literature review in the previous section shows that alternative methods are required. One possibility is to define IVIFSs using Gaussian membership functions and develop distance and similarity measures between IVIFS-GMFs.

Thus, the objectives of this research included the following:

- define IVIFSs using Gaussian membership functions (IVIFS-GMFs).

- derive distance measures for IVIFS-GMFs.

- define similarity measures for IVIFS-GMFs. 


\section{Preliminaries}

In this section, we provide theoretical background material for developing the new distance measures.

Definition 4.1. Gaussian membership functions (GMFs).

In fuzzy logic, the function $f(x ; \sigma, c)=e^{-\frac{1}{2}\left(\frac{x-c}{\sigma}\right)^{2}}$ defines a Gaussian membership function, where $\mathrm{c}$ is the centre point of the membership function and $2 \sigma$ indicates the span of the curve.

Definition 4.2. Interval-valued intuitionistic fuzzy set (IVIFS).

Let $\mathrm{X}$ be a universal set and let $[I]$ denote the set of all closed subintervals of $[0,1]$. $\mathrm{A}$ set $\mathrm{A}$ in $\mathrm{X}$ is said to be an IVIFS if $A=\left\{\left\langle x, \mu_{A}(x), v_{A}(x)\right\rangle / x \in X\right\}$ where $\mu_{A}, v_{A}: X \rightarrow[I], \quad \mu_{A}(x)=\left[\mu_{L}(x), \mu_{U}(x)\right], \quad v_{A}(x)=\left[v_{L}(x), v_{U}(x)\right]$, And $0 \leq \mu_{U}(x)+$ $v_{U}(x) \leq 1$ for any $x \in X[2]$.

\subsection{Bhattacharya Distance Between Two Distributions}

The Bhattacharya distance gives a measure of the similarity between two probability distributions, and the Bhattacharya coefficient gives a measure of the overlap between two Gaussian distributions.

Definition 4.3. Bhattacharya distance.

The Bhattacharya distance is defined as

$$
D_{B}(p, q)=\frac{1}{4} \ln \left(\frac{1}{4}\left(\frac{\sigma_{p}^{2}}{\sigma_{q}^{2}}+\frac{\sigma_{q}^{2}}{\sigma_{p}^{2}}+2\right)\right)+\frac{1}{4}\left(\frac{\left(\mu_{p}-\mu_{q}\right)^{2}}{\sigma_{p}^{2}+\sigma_{q}^{2}}\right)
$$

where $\mathrm{p}$ and $\mathrm{q}$ are two distributions, $\sigma_{p}^{2}$ and $\sigma_{q}^{2}$ are the variances of the distributions $\mathrm{p}$ and $\mathrm{q}$, respectively, and $\mu_{p}$ and $\mu_{q}$ are the means of the distributions $\mathrm{p}$ and $\mathrm{q}$, respectively [3].

Definition 4.4. Bhattacharya coefficient (BC).

The Bhattacharya coefficient is defined using the Bhattacharya distance and is given by

$$
\begin{gathered}
D_{B}(p, q)=-\ln (B C(p, q)) ; \\
B C(p, q)=e^{-D_{B}(p, q)} .
\end{gathered}
$$

It can be shown that $0 \leq B C \leq 1$ and $0 \leq D_{B} \leq \infty$.

The Bhattacharya distance does not satisfy the triangle inequality.

Hence, the Hellinger distance $\sqrt{1-e^{-D_{B}(p, q)}}$, which does satisfy the triangle inequality, was chosen as the distance metric [3]. 
Definition 4.5. Bhattacharya angle [3].

The Bhattacharya angle gives the angle between two probability measures and is defined as

$$
\Delta(p, q)=\arccos e^{-D_{B}(p, q)} .
$$

\section{Interval-Valued Intuitionistic Gaussian Membership Functions}

In this section, we present the IVIFS with Gaussian membership functions. The following distance measures were developed for IVIFS-GMFs:

- the Bhattacharya distance for IVIFS-GMFs and cut sets on an IVIGMF.

- an overlap measure for IVIFS-GMFs.

- a measure of the angle between two IVIFS-GMFs.

Definition 5.1. Interval-valued intuitionistic Gaussian membership function (Fig. 1).

The membership, non-membership, and hesitancy functions are, respectively,

$$
\begin{aligned}
& \mu_{\tilde{A}_{G}}(x)=\left[\mu_{\tilde{A}_{G}}^{L}(x), \mu_{\tilde{A}_{G}}^{U}(x)\right], \\
& v_{\tilde{A}_{G}}(x)=\left[v_{\tilde{A}_{G}}^{L}(x), v_{\tilde{A}_{G}}^{U}(x)\right], \\
& \pi_{\tilde{A}_{G}}(x)=\left[\pi_{\tilde{A}_{G}}^{L}(x), \pi_{\tilde{A}_{G}}^{U}(x)\right] .
\end{aligned}
$$

and satisfy the following conditions:

Membership:

$$
\mu_{\tilde{A}_{G}}^{L}(x)=u^{-} \times e^{-\frac{1}{2}\left(\frac{x-c}{\sigma}\right)^{2}}, 0<u<1, \mu_{\tilde{A}_{G}}^{U}(x)=u^{+} e^{-\frac{1}{2}\left(\frac{x-c}{\sigma}\right)^{2}}
$$

Non-Membership:

$$
v_{\tilde{A}_{G}}^{L}(x)=v \times\left(1-\mu_{A L}\right), v_{\tilde{A}_{G}}^{U}(x)=v \times\left(1-\mu_{A U}\right), 0<v<u^{+}<1 ;
$$

Hesitancy:

$$
\pi_{\tilde{A}_{G}}^{L}(x)=1-\mu_{\tilde{A}_{G}}^{L}(x)-v_{\tilde{A}_{G}}^{L}(x), \pi_{\tilde{A}_{G}}^{U}(x)=1-\mu_{\tilde{A}_{G}}^{U}(x)-v_{\tilde{A}_{G}}^{U}(x) .
$$


Definition 5.2. Distance, overlap, and angle measures for IVIFS-GMFs.

The Bhattacharya distance for IVIFS-GMFs can be defined as

$$
\begin{gathered}
\tilde{d}_{B h}(A, B)\left(x_{i}\right)=\frac{1}{8}\left[\begin{array}{l}
\ln \frac{1}{4}\left\{\left(\frac{\sigma_{A}^{2}}{\sigma_{B}^{2}}+\frac{\sigma_{B}^{2}}{\sigma_{A}^{2}}+2\right)\right\}+\frac{1}{\left(\sigma_{A}^{2}+\sigma_{B}^{2}\right)} \times \\
{\left[\begin{array}{l}
\left(c_{A}-c_{B}\right)^{2} \\
+\left\{\left(\mu_{A L}\left(x_{i}\right)-\mu_{B L}\left(x_{i}\right)\right)+\left(\mu_{A U}\left(x_{i}\right)-\mu_{B U}\left(x_{i}\right)\right)\right\}^{2} \\
+\left\{\left(v_{A L}\left(x_{i}\right)-v_{B L}\left(x_{i}\right)\right)+\left(v_{A U}\left(x_{i}\right)-v_{B U}\left(x_{i}\right)\right)\right\}^{2} \\
+\left\{\left(\pi_{A L}\left(x_{i}\right)-\pi_{B L}\left(x_{i}\right)\right)+\left(\pi_{A U}\left(x_{i}\right)-\pi_{B U}\left(x_{i}\right)\right)\right\}^{2}
\end{array}\right]}
\end{array}\right] \\
\tilde{D}_{B h}(A, B)=\frac{1}{8} \sum_{i=1}^{n} \tilde{d}_{B h}(A, B)\left(x_{i}\right)
\end{gathered}
$$

The overlap of two IVIFS-GMFs can be defined as

$$
C_{B h}(p, q)=e^{-D_{B h}(p, q)}
$$

The Bhattacharya angle between two IVIFS-GMFs is given by

$$
\Delta(A, B)=\arccos C_{B h}(A, B)
$$

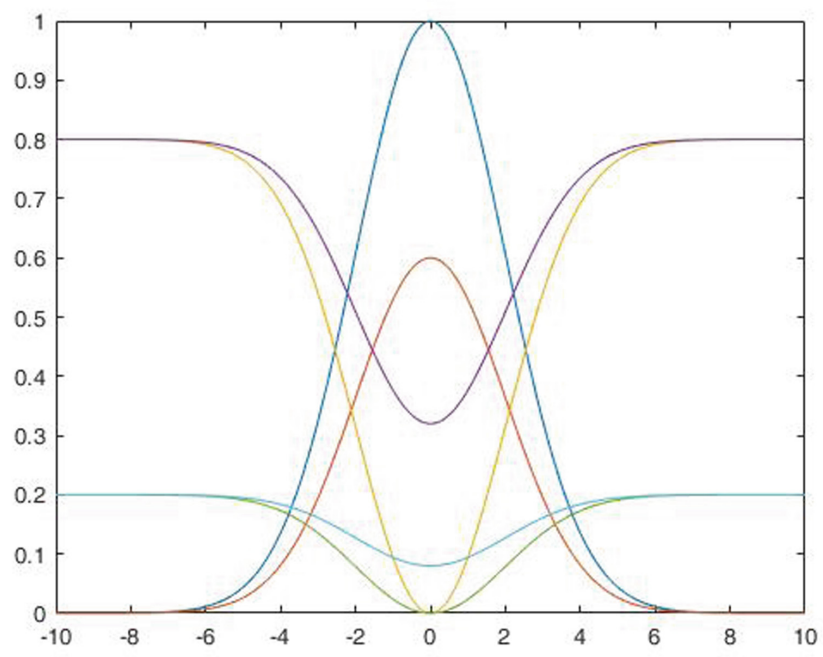

Fig. 1. IVIFS with Gaussian membership functions for $c=0, \sigma=2$

The Bhattacharya distance for IVIFS-GMFs clearly satisfies the requirements for a distance metric. 


\section{Example: Similarities in Two Individuals Using Genetic Brain Profiling and IVIFS-GMF}

Genetic Brain Profiling (GBP) is a unique assessment tool that provides individuals with greater knowledge of their traits and their most beneficial environment. This tool identifies innate talents, which assists in developing the full potential of an individual and identifies the most beneficial educational system and environment for an individual. GBP identifies possible behavioural and learning difficulties and academic strengths and weaknesses. In addition, GBP provides information on how an individual receives social and educational information. Furthermore, GBP identifies interpersonal communication styles, the causes of stress, and an individual's tendencies for handling stress. GBP is based on the principles of the behavioural sciences and applied psychology. This approach provides a procedure to map the learning, cognitive, and action preferences of an individual and their effects on an individual's behaviour. GBP analyses fingerprints to identify an individual's natural abilities, talents, and personality traits. Learning style, personality, and aptitude can be identified in an individual as early as age two with GBP, and this information can give parents a better understanding of their child.

\section{Materials, Method and Result}

To test IVIFS-GMFs with the Bhattacharya distance using GBP analysis as an example, the fingerprints of two individuals were obtained and the profiles were created. Educational performance data were chosen as test samples.

Table 1 lists the performance in three levels of formal learning, namely, primary, secondary, and tertiary, or university, education. The performance data are comfort levels expressed as a percentage at each level.

Table 1. Performance at three levels of formal learning

\begin{tabular}{l|l|l|l}
\hline Education level & Primary & Secondary & University \\
\hline Individual 1 & 30.51 & 36.5 & 32.99 \\
\hline Individual 2 & 33.49 & 31.86 & 34.65 \\
\hline
\end{tabular}

Table 2 gives the scores for the primary sensory learning styles. These scores indicate the comfort levels of an individual in using their various physical senses. Scores are obtained for three styles of learning: kinaesthetic, auditory, and visual. An analysis of these scores indicates the degree to which an individual is reflective, affective, and cognitive; the analysis results are included in Table 2.

Table 3 provides the intelligence profiles for the two test samples, where several types of intelligence such as logic, verbal, nature, and music were assessed.

Table 4 provide the four DISC factors, which summarize an individual's profile. DISC is an acronym for dominance, influence, steadiness, and compliance. 
Table 2. Primary sensory learning styles.

\begin{tabular}{l|l|l|l}
\hline Learning & Kinaesthetic & Auditory & Visual \\
\hline Individual 1 & 26.98 & 29.89 & 43.13 \\
\hline Individual 2 & 32.77 & 33.45 & 33.78 \\
\hline Domains & Reflective & Affective & Cognitive \\
\hline Individual 1 & 40 & 30 & 30 \\
\hline Individual 2 & 20 & 70 & 10 \\
\hline
\end{tabular}

Table 3. Genetic Intelligence profiles of test samples.

\begin{tabular}{l|l|c|l|c|c}
\hline Intelligence category & Logic & Verbal & Self & People & Body \\
\hline Individual 1 & 12.5 & 12.7 & 11.6 & 9.4 & 9.7 \\
\hline Individual 2 & 10.2 & 8.9 & 10.2 & 10.7 & 10.7 \\
\hline Intelligence category & Nature & Music & Picture & Cosmic & \\
\hline Individual 1 & 12.6 & 9.7 & 9.9 & 11.8 & \\
\hline Individual 2 & 12.2 & 13.1 & 14.7 & 9.1 & \\
\hline
\end{tabular}

Table 4. Genetic behaviour profiles.

\begin{tabular}{l|l|l|l|l|l|l}
\hline GBP & Persistence & Sensitivity & $\begin{array}{l}\text { Self } \\
\text { confidence }\end{array}$ & Thoughtfulness & Enthusiasm & $\begin{array}{l}\text { Self- } \\
\text { motivation }\end{array}$ \\
\hline Individual 1 & 1.16 & 0.87 & 0.91 & 1.1 & 1.05 & 1.17 \\
\hline Individual 2 & 1.39 & 0.72 & 0.97 & 1.03 & 1.35 & 0.81 \\
\hline $\begin{array}{l}\text { Intelligence } \\
\text { category }\end{array}$ & Patience & Cooperativeness & Independence & Friendliness & Accuracy & Efficiency \\
Individual 1 & 0.86 & 0.74 & 1.35 & 0.78 & 0.95 & 1.28 \\
\hline Individual 2 & 1.23 & 0.89 & 1.13 & 1.2 & 0.75 & 0.83 \\
\hline
\end{tabular}

The DISC factors provide a useful technique for extracting information that describes the relationships between various pairs of factors.

\section{Experimental Results}

The mean and the variance of the data were calculated, and the Bhattacharya distance, coefficient, and angle were obtained. The results are shown in Table 5.

Table 5. Similarity evaluation of GBPs.

\begin{tabular}{l|l|l|l|l}
\hline Profiles & Bhattacharya distance & Overlap & \multicolumn{2}{|l}{$\begin{array}{l}\text { Bhattacharya } \\
\text { angle }\end{array}$} \\
\cline { 3 - 5 } & & & Radians & Degrees \\
\hline Education & 0.4952 & 0.6094 & 0.9154 & 52.45 \\
\hline Learning & 4.9977 & 0.0068 & 1.564 & 89.61 \\
\hline Domains & 2.1155 & 0.1206 & 1.4499 & 83.07 \\
\hline Intelligence profile & 0.1055 & 0.89 & 0.4513 & 25.86 \\
\hline GBP & 0.1396 & 0.8697 & 0.5161 & 29.57 \\
\hline
\end{tabular}


Table 5 clearly shows that the two individuals are most similar in their intelligence and behaviour profiles and least similar in their learning styles and domains.

The results were found to be consistent with the profiler's interpretation of the results.

\section{Conclusion and Future Direction}

Interval-valued intuitionistic fuzzy sets have been applied to problems in distancebased pattern recognition and in evaluating similarities between samples. In this research, we defined interval-valued intuitionistic Gaussian membership functions and defined new measures of the distance, the overlap, and the angle between two IVIFSGMFs. The proposed method was used to analyse samples of Genetic Brain Profiling (GBP) to determine the similarity between two individuals. IVIFS-GMFs can be applied in image segmentation, pattern recognition, and MCDM problems where Bhattacharya angle and distance are widely used. Further, this formula can be used to study the Gaussian distribution curve of the COVID 19 infected countries and the uncertainty areas can be addressed.

Acknowledgements. The author is sincerely grateful to Mrs. Rekha Hariprawin, SARE Image Consultancy, Chennai, for providing the GBP samples, which were provided in collaboration with MIDNA Global (www.midnaglobal.com), India.

\section{References}

1. Adak, A.K., Bhowmik, M., Pal, M.: Interval cut-set of generalised interval-valued intuitionistic fuzzy sets. Int. J. Fuzzy Syst. Appl. 2, 35-50 (2012)

2. Atanassov, K., Gargov, G.: Interval-valued intuitionistic fuzzy sets. Fuzzy Sets Syst. 31, 343-349 (1989)

3. Bhattacharyya, A.: On a measure of divergence between two statistical populations defined by their probability distributions. Bull. Calcutta Math. Soc. 35, 99-109 (1983)

4. Meng, F., Chen, X.: Entropy and similarity measure for Atanassov's interval valued intuitionistic fuzzy sets and their application. Fuzzy Optim. Decis. Making 15, 75-101 (2016)

5. Singh, P.: A new method on measure of similarity between interval-valued intuitionistic fuzzy sets for pattern recognition. J. Appl. Comput. Math. 1, 1-5 (2012)

6. Wei, C.P., Wang, P., Zhang, Y.Z.: Entropy, Similarity measure of interval valued intuitionistic fuzzy sets and their applications. Inf. Sci. 181, 4273-4286 (2011)

7. Yuan, X.H., Li, H.X.: Cut sets on interval-valued intuitionistic fuzzy sets. In: Proceedings of the 6th International Conference on Fuzzy Systems and Knowledge Discovery, pp. 167-171 (2009)

8. Yuan, X., Li, H., Sun, K.: The cut sets, decomposition theorems and representation theorems on intuitionistic fuzzy sets and interval-valued fuzzy sets. Sci. China Inf. Sci. 54, 91-110 (2010)

9. Zeshui, X.: On Similarity measures of interval-valued intuitionistic fuzzy sets and their applications to pattern recognition. J. Southeast Univ. 23, 139-143 (2007) 
10. Zhang, Q.S., Yao, H.X., Zhang, Z.H.: Some similarity measures of interval valued intuitionistic fuzzy sets and application to pattern recognition. Appl. Mech. Mater. 44-47, 3888-3892 (2011)

11. Zhang, Y., Ma, P., Su, X.: Pattern recognition using interval-valued intuitionistic fuzzy set and its similarity degree. In: Proceedings of the IEEE International Conference on Intelligent Computing and Intelligent Systems, Shanghai, China (2009)

12. Zhang, Z., Wang, M., Hu, Y., Ye, Y., Li, Y.: A dynamic interval-valued intuitionistic fuzzy sets applied to pattern recognition. Math. Probl. Eng. 2013, 1-16 (2013) 